\title{
SELEÇÃO DE PLANTAS COM ATIVIDADE ANTICOLINASTERASE PARA TRATAMENTO DA DOENÇA DE ALZHEIMER
}

Maria Teresa Salles Trevisan* e Fábio Vanderlan Viana Macedo

Departamento de Química Orgânica e Inorgânica, Centro de Ciências, Universidade Federal do Ceará, CP 12200, 60021-970

Fortaleza - CE, Brasil

Michiel van de Meent, In Kyung Rhee e Rob Verpoorte

Division of Pharmacognosy, Leiden University, Gorlaeus Laboratories, P.O. Box 9502, 2300 RA Leiden. The Netherlands

Recebido em 12/12/01; aceito em 5/11/02

\begin{abstract}
SCREENING FOR ACETYLCHOLINESTERASE INHIBITORS FROM PLANTS TO TREAT ALZHEIMER'S DISEASE. Alzheimer's disease (AD) is a neurodegenerative disease characterized by cognite impairment and personality changes. The development of drugs for the treatment of the cognitive deficits of AD has focused on agents which counteract loss in cholinergic activities. These symptons of AD have been successfully treated with acetylcholinesterase (AchE) inhibitors (eg. galanthamine). There still is great interest in finding better AchE inhibitors. We use Ellmann's microplate assay and silica gel thin-layer chromatography (TLC) to screen natural products from plants as new sources of AchE inhibitors.
\end{abstract}

Keywords: Alzheimer disease; plants; cholinesterase inhibitors.

\section{INTRODUÇÃO}

Na última década, a maneira de se pesquisarem novos compostos biologicamente ativos sofreu grandes mudanças, principalmente devido aos avanços tecnológicos. A indústria farmacêutica tem um papel importante no desenvolvimento de novos métodos, os quais podem propiciar, de forma mais rápida, o surgimento de novos medicamentos no mercado ${ }^{1}$.

Um dos mais importantes fatores para o sucesso no descobrimento de um fármaco novo é a diversidade química dos compostos a serem selecionados, cujas fontes podem ser: compostos sintéticos, produtos naturais ou química combinatória. Entre estas possibilidades, os produtos naturais são considerados como uma das maiores fontes de diversidade química ${ }^{2}$.

A natureza é uma fonte de inestimável riqueza de novos medicamentos. Nos EUA e Inglaterra, aproximadamente $25 \%$ dos compostos ativos, nas prescrições atuais, foram identificados em plantas superiores $^{3}$.

A avaliação biológica ${ }^{1}$ foi uma das mais significantes mudanças na área de produtos naturais nos últimos anos. O entendimento dos mecanismos da doença, acompanhado do aumento de testes com receptores e enzimas disponíveis, permitiram o desenvolvimento de sistemas eficientes e rápidos de bioensaios. Dessa forma, um bom sistema permite selecionar milhares de amostras em poucos dias ${ }^{1,4}$.

A modernização dos ensaios permitiu a utilização de enzimas, receptores, DNA, entre outros alvos, para avaliação rápida de grandes quantidades de amostras. Dentre os bioensaios rápidos e sensíveis, a utilização da enzima acetilcolinesterase é uma alternativa para a detecção e seleção de amostras com ação anticolinesterase.

Entre os indivíduos idosos, ressaltam-se as anormalidades decorrentes da disfunção do sistema nervoso, como deficiências relativas à memória, à capacidade intelectual, à coordenação motora, ao equilíbrio, à postura, etc.

A demência, embora possa ocorrer nas idades jovens por enfermidades cerebrais variadas, está ligada ao fenômeno de envelheci-

* e - mail: trevisan@ufc.br mento cerebral, acometendo indivíduos com idade superior a 65 anos. Admite-se que 10 a $15 \%$ destes indivíduos apresentam alguma forma de demência e, deste grupo, 50 a $60 \%$ sofrem de uma doença degenerativa de causa não totalmente conhecida denominada doença de Alzheimer ${ }^{5-8}$.

A doença de Alzheimer está associada com "déficits" dos diversos neurotransmissores cerebrais, como a acetilcolina, a noradrenalina e a serotonina ${ }^{8}$. O tratamento sintomático da doença de Alzheimer envolve primariamente a restauração da função colinérgica. Sugerese, portanto, que uma elevação no nível da acetilcolina poderia ser útil para melhorar um dos sinais da doença, a deficiência de aprendizagem 9 . Até a presente data, os inibidores da colinesterase demonstraram a maior eficiência no tratamento clínico na doença de Alzheimer.

O uso de medicamentos complementares, como extratos de plantas, em terapia para doenças do cérebro, varia de acordo com as diferenças culturais. Na medicina ortodoxa ocidental, em contraste com a oriental (a medicina chinesa, por exemplo), propriedades farmacológicas de plantas relacionadas ao aumento de memória/ aprendizagem não são investigadas, com exceção dos gincolídeos, cuja ação neuroprotetora e atividades colinérgicas foram consideradas relevantes e comprovadamente eficazes. Ressalte-se, ainda, que o uso dos gincolídeos não apresentou efeitos colaterais indesejáveis no tratamento da doença de Alzheimer ${ }^{10}$.

Companhias farmacêuticas vêm apoiando fortemente a pesquisa e o desenvolvimento de fármacos que aumentem a capacidade cognitiva. Assim, a pesquisa acerca da possibilidade de que a elevação no nível da acetilcolina seja útil para melhorar os sinais de deficiência de aprendizagem ${ }^{10}$ apresenta-se como algo a ser incentivado. Os inibidores da colinesterase, os quais aumentam a função colinérgica central, são utilizados no tratamento da doença de Alzheimer. Esses mesmos benefícios terapêuticos poderiam ser úteis para crianças com retardamento mental ${ }^{11}$.

Os fármacos inibidores da acetilcolinesterase, usualmente disponíveis no mercado, são Cognex (tacrina) e Exelon (rivastigmina) e apresentam, hoje, preços bastante onerosos, R\$ 130,00 e R\$105,00, respectivamente. Fármaco como Reminyl (galantanina), que recente- 
mente foi aprovado pelo FDA (fevereiro de $2001^{12}$ ), atualmente disponível no mercado brasileiro, é vendido a $\mathrm{R} \$ 316,00$. Portanto, pela situação apresentada, seria de grande interesse para a população a disponibilização de métodos alternativos para o tratamento da doença de Alzheimer, como a utilização de plantas medicinais específicas.

Este trabalho descreve o estudo de extratos (aquoso, hidroalcoólico, etanólico, metanólico, acetato de etila, clorofórmico, hexânico) de plantas pertencentes a vários gêneros e famílias (Amburana, Anacardium, Auxemma, Bauhinia, Bowdicha, Cecropia, Cordia, Croton, Dalechampia, Egletes, Lippia, Lonchocarpus, Mimosa, Momordica, Paullinia, Philodendron, Plathymenia, Plathymiscium, Protium, Pterodon, Simarouba, Solanum, Triphasia, Vanilosmopsis, Verbezina, Vitex) que poderiam inibir a atividade da enzima acetilcolinesterase.

\section{RESULTADOS E DISCUSSÃO}

O critério de escolha das plantas baseou-se nos ensaios dos respectivos extratos, já em estudos no grupo de Produtos Naturais do Departamento de Química Orgânica e Inorgânica da UFC. Objetivase selecionar plantas cujos extratos inibam a atividade da acetilcolinesterase e, em uma etapa posterior, utilizar bioensaio para isolar e caracterizar princípios ativos anticolinesterásicos.

Os resultados dos extratos ensaiados estão apresentados na Tabela 1 .

A maioria dos extratos (42) foi testada em uma concentração de $1.0-2.5 \mathrm{mg} / \mathrm{mL}$.

Alguns extratos, como o da Verbezina (flores) e do Vanilosmopsis (flores), ambos em hexano, não dissolveram em metanol, fato que limitou a análise por meio do ensaio na microplaca. Os ensaios, nestes extratos, foram feitos somente em CCD e deram resultados negativos.

Extratos cuja inibição enzimática foi maior ou igual a 50\% são considerados candidatos a futuros fracionamentos. Pela análise da Tabela 1, pode-se considerar que $20,7 \%$ dos extratos testados seriam candidatos a um fracionamento bio-guiado, para isolamento de princípios ativos capazes de inibir a enzima acetilcolinesterase.

Por meio da comparação dos resultados obtidos com os dois tipos de ensaio (microplaca e CCD) e considerando que extratos com menos de $30 \%$ de inibição enzimática sejam falsos positivos, podese concluir que a confiabilidade do ensaio via CCD é de $81,1 \%$. A análise da Tabela 1 mostra 17,2\% de falsos positivos e somente 1,7\% de falsos negativos, o que demonstra ser esse um método confiável, rápido e barato para bio-guiar fracionamentos futuros.

Pode-se confirmar halos de inibição, que ocorrem devido à hidrólise espontânea da acetiltiocolina em CCD, e que levaria aos falsos positivos, colocando-se a mistura enzima $\mathrm{AChE}$ e acetiltiocolina a $37{ }^{\circ} \mathrm{C}, 20 \mathrm{~min}$, antes de aplicá-la na placa.

A presença de taninos em extratos brutos pode levar a falsos positivos, não pela inibição, mas por promover a desnaturação da enzima acetilcolinesterase. Portanto, tem-se de considerar sempre que testes in vitro apenas auxiliam na otimização da seleção e não eliminam, de forma alguma, os testes in vivo.

A presença de taninos e compostos fenólicos poderia ser verificada por análise cromatográfica. Assim, se os halos de inibição estiverem na origem, ou seja, nos compostos mais polares, provavelmente haja taninos e compostos fenólicos presentes no extrato, o que poderia ser confirmado pelo teste para fenóis e taninos ${ }^{13}$.

\section{CONCLUSÕES}

A análise da Tabela 1 mostra plantas bastante promissoras para isolamento e caracterização de compostos:
Paullinia cupana (guaraná), cujo extrato inibiu a enzima em $65 \%$. Estudos realizados mostraram que, após uma administração única (3,0 e $30 \mathrm{mg} / \mathrm{kg}$ ) ou crônica $(0,3 \mathrm{mg} / \mathrm{ml})$ de pó da semente de guaraná, reverteu-se parcialmente o efeito da escopolamina indicando um efeito positivo na aquisição de memória ${ }^{14}$;

Amburana cearensis (cumaru), cujo extrato inibiu a enzima em $100 \%$. Planta rica em cumarina ${ }^{15}$, em Angelica gigas, seu fracionamento bio-guiado, utilizando-se a atividade inibitória da AchE, levou ao isolamento e identificação de uma nova cumarina e ao isolamento de mais 11 cumarinas já descritas na literatura ${ }^{16}$. Portanto, por meio desse procedimento, pode-se alcançar o isolamento e a identificação de novos inibidores da AchE;

Lippia sidoides, com inibições que variam de 7 a 77\%, dependendo do extrato e da parte da planta utilizada no ensaio. Esse teste mostrou-se útil para o procedimento bio-guiado e, no caso da Lippia, fez-se a escolha do extrato das folhas em metanol para fracionamentos posteriores.

As plantas referidas são apenas alguns dos exemplos do potencial desse bioensaio para o isolamento e caracterização de novos inibidores da AchE.

\section{PARTE EXPERIMENTAL}

\section{Procedimento geral para a preparação dos extratos das plantas}

O material vegetal da planta após ser pulverizado mecanicamente, foi submetido à extração exaustiva, utilizando-se os solventes indicados na Tabela 1. Após destilação do solvente, foi obtido o extrato bruto utilizado nos ensaios. A identificação das espécies foi realizada por botânicos do Herbário "Prisco Bezerra", do Departamento de Biologia da UFC, onde se encontram depositadas as exsicatas.

\section{Medida da atividade de acetilcolinesterase}

Prepararam-se as seguintes soluções: (1) $50 \mathrm{mM}$ Tris/HCl pH 8; (2) $50 \mathrm{mM}$ Tris/ $\mathrm{HCl} \mathrm{pH} 8$, contendo $0,1 \%$ de Albumina sérica bovina (BSA) fração $\mathrm{V}$; (3) $50 \mathrm{mM}$ Tris/ $\mathrm{HCl} \mathrm{pH} 8$, contendo $0,1 \mathrm{M}$ de $\mathrm{NaCl}$ e $0,02 \mathrm{M}$ de $\mathrm{MgCl}_{2} \cdot 6 \mathrm{H}_{2} \mathrm{O}$; (4) $3 \mathrm{mM}$ de ácido 5,5'-ditiobis-[2nitrobenzóico] (DTNB ou reagente de Ellman), (5) $15 \mathrm{mM}$ de iodeto de acetilcolina (ACTI), (6) $1 \mathrm{mM}$ de ácido 5,5'-ditiobis-[2nitrobenzóico] (DTNB ou reagente de Ellman) e (7) $1 \mathrm{mM}$ de iodeto de acetiltiocolina (ACTI).

A enzima AChE liofilizada foi dissolvida na solução tampão (1) para fazer uma solução estoque $1000 \mathrm{U} / \mathrm{mL}$, deixando-se na solução por $20 \mathrm{~min}$, depois sob agitação por mais um período de 10-15 min, para obtenção de uma solução homogênea. Para as diluições posteriores, utilizou-se a solução tampão (2).

\section{Ensaio na microplaca}

Para a reação enzimática no ensaio de microplaca, utilizou-se uma leitora de microplaca Bio-Rad, modelo 3550 UV, absorbância a $405 \mathrm{~nm}$, baseado no método de Ellman ${ }^{17,18}$. A enzima hidrolisou o substrato acetiltiocolina, gerando como produto a tiocolina, que reage com o reagente de Ellman, produzindo 2-nitrobenzoato-5mercaptotiocolina e 5-tio-2-nitrobenzoato, que podem ser detectados a $405 \mathrm{~nm}$. Nas 96 cavidades da placa, adicionaram-se $25 \mu \mathrm{L}$ de (5); $125 \mu \mathrm{L}$ de (4); $50 \mu \mathrm{L}$ de (2); $25 \mu \mathrm{L}$ da amostra a ser analisada, dissolvida em $\mathrm{MeOH}$, diluída 10 vezes em (1); mediu-se a absorbância a cada 13 s por 5 vezes. Adicionou-se $25 \mu \mathrm{L}$ da enzima $(0,22 \mathrm{U} / \mathrm{mL})$; mediu-se novamente a absorbância a cada 13 s por 8 vezes. Calculou-se a velocidade das reações utilizando-se o software Microplate Manager version 4.0 (Bio-Rad Laboratories). Corrigiram-se os au- 
Tabela 1. Inibição da atividade da enzima acetilcolinesterase por extratos brutos de plantas ensaiados na microplaca (\% de inibição) e em cromatografia de camada delgada (CCD)

\begin{tabular}{|c|c|c|c|}
\hline Plantas & Concentração extrato $(\mathrm{mg} / \mathrm{mL})$ & Microplaca (\% Inibição) & CCD \\
\hline 1. Amburana cearensis (casca do caule) (5) & 2,3 & 100 & + \\
\hline 2. Anacardium occidentale L. (casca) (5) & 2,3 & 09 & - \\
\hline 3. Auxemma glazioviana (caule) (2) & 2,5 & 43 & + \\
\hline 4. Auxemma glazioviana (caule) (3) & 0,6 & 100 & - \\
\hline 5. Bauhinia chyilantha (folhas) (9) & 1,0 & 03 & - \\
\hline 6. Bowdicha virigiloide (caule) (1) & 0,6 & 0 & - \\
\hline 7. Bowdicha viroigiloide (caule) (4) & 1,5 & 0 & + \\
\hline 8. Cecropia pachystachya (folhas) (5) & 1,1 & 0 & + \\
\hline 9. C. anguria (caule) (1) & 1,8 & 0 & - \\
\hline 10. Cordia piauhiensis (raiz) (2) & 2,7 & 0 & - \\
\hline 11. Cordia piauhiensis (raiz) (4) & 3,9 & 0 & - \\
\hline 12. Cordia piauhiensis (caule) (5) & 1,7 & 0 & + \\
\hline 13. Croton urucunama $(7)$ & 1,5 & 39 & + \\
\hline 14. Dalechampia fernanendesii (flores, frutos) (5) & 0,6 & 10 & - \\
\hline 15. Egletes viscosa (capítulos florais) (2) & 1,3 & 28 & + \\
\hline 16. Lippia alba (folhas) (1) & 0,9 & 0 & - \\
\hline 17. Lippia alba (folhas) (5) & 2,1 & 12 & + \\
\hline 18. Lippia sidoides (Talos) (4) & 1,5 & 31 & + \\
\hline 19. Lippia sidoides (caule sem cascas) (5) & 2,0 & 46 & + \\
\hline 20. Lippia sidoides (cascas) (3) & 1,4 & 07 & - \\
\hline 21. Lippia sidoides (folhas) (4) & 2,8 & 60 & + \\
\hline 22. Lippia sidoides (folhas) (5) & 2,2 & 77 & + \\
\hline 23. Lippia sidoides (folhas) (6) & 2,3 & & - \\
\hline 24. Lonchocarpus sericeus (casca do caule) (5) & 2,5 & 0 & - \\
\hline 25. Lonchocarpus sericeus (lenho da raiz) (5) & 2,7 & 13 & - \\
\hline 26. Mimosa acustipula Jurema (casca do caule)(5) & 1,8 & 39 & - \\
\hline 29. Mormodica charantia (talos) (1) & 1,5 & 0 & - \\
\hline 30. Mormodica charantia (talos) (5) & 0,8 & 0 & $+/-$ \\
\hline 31. Paullinia cupana (5) & 1,5 & 65 & + \\
\hline 32. Philodendron Imbé (folhas) (10) & 1,4 & 0 & - \\
\hline 33. Philodendron Imbé (folhas) (3) & 1,5 & 0 & $+/-$ \\
\hline 34. Philodendron Imbé (folhas) (4) & 2,3 & 29 & + \\
\hline 35. Philodendron Imbé (caule) (11) & 2,5 & 0 & - \\
\hline 36. Philodendron Imbé (folhas) (F70-72) & 2,5 & 0 & - \\
\hline 37. Plathymenia reticulata (casca do caule) (2) & 1,4 & 88 & - \\
\hline 38. Plathymiscium floribundum (cerne) (5) & 2,8 & 72 & + \\
\hline 39. Protium heptaphyllum (resina) (1) & 3,3 & 25 & + \\
\hline 40. Protium heptaphyllum (resina) (12) & 17 & 48 & + \\
\hline 41. Pterodon polygalaeflorus (semente) (3) & 2,3 & 12 & - \\
\hline 42. Pterodon polygalaeflorus (semente) (4) & 1,5 & 05 & - \\
\hline 43. Pterodon polygalaeflorus (semente) (5) & 2,0 & 05 & - \\
\hline 44. Simarouba versicolor (fruto) (5) & 1,5 & 07 & - \\
\hline 45. Solanum asperum (folhas) (5) & 1,4 & 60 & + \\
\hline 46. Triphasia trifolia (folhas) (1) & 1,1 & 0 & + \\
\hline 47. Triphasia trifolia (folhas) (2) & 1,4 & 50 & + \\
\hline 48. Triphasia trifolia (folhas) (3) & 1,8 & 0 & + \\
\hline 49. Triphasia trifolia (folhas) (4) & 2,1 & 53 & + \\
\hline 50. Vanilosmopsis arborea (flores) (1) & & & - \\
\hline 51. Vanilosmopsis arborea (8) & 1,2 & 37 & + \\
\hline 52. Vanilosmopsis arborea (5) & 1,2 & 50 & + \\
\hline 53. Verbezina diversifolia (flores) (1) & & & - \\
\hline 54. Verbezina diversifolia (flores) (5) & 1,6 & 0 & + \\
\hline 55. Vitex agnus castus (folhas) (4) & 1,7 & 12 & + \\
\hline 56. Vitex agnus castus (frutos) (5) & 1,5 & 18 & - \\
\hline 57. Velame A (5) & 2,5 & 40 & + \\
\hline 58. Velame B (5) & 1,6 & 65 & + \\
\hline
\end{tabular}

+, inibição; -, não inibição; *solvente utilizado na extração: (1) hexano, (2) clorofórmio, (3) acetato de etila, (4) metanol, (5) etanol, (6) água, (7) latex liofilizado, (8) diclorometano: metanol (1:1), (9) hidroalcoólico, (10) hexano: clorofórmio (80\%), (11) diclorometano: acetato de etila (1:1), (12) óleo essencial 
mentos em absorbância, devido à hidrólise espontânea, por meio da subtração da velocidade da reação antes de se adicionar a enzima com a velocidade da reação depois de se adicionar a enzima. A porcentagem de inibição calculou-se pela comparação das velocidades das amostras em relação ao branco (10\% MeOH no tampão 1).

\section{Ensaio em cromatografia de camada delgada (CCD)}

Aplicaram-se as amostras (1,5 - 2,5 $\mu \mathrm{L})$ em CCD, DC-Alufolien, Silicagel 60 F254, 0,2 mm Merck. Borrifou-se a placa com as soluções $(6)+(7)$, deixando 3 min. Após secar, borrifou-se a enzima 3 $\mathrm{U} / \mathrm{mL}$ e, em $10 \mathrm{~min}$, apareceu a coloração amarela, mas onde houve inibição da enzima, observou-se um halo branco ${ }^{18}$. Em 20 - 30 min a coloração desapareceu.

\section{AGRADECIMENTOS}

Os autores agradecem ao $\mathrm{CNPq}$ pela grande ajuda de F. V. V. Macedo - bolsista de IC - na realização desse trabalho. À Funcap, pela passagem concedida para a realização dos ensaios na Universidade de Leiden, Holanda. Aos professores da Pós- Graduação de Química Orgânica do Depto. de Química Orgânica e Inorgânica da UFC: A. M. C. Arriaga, E. R. Silveira, F. Monte, M. Andrade Neto, M. Z. B. Bezerra, T. L. G. de Lemos, Y. B. M. Pouliquen, O. D. L. C. Pessoa, por fornecerem os extratos que foram analisados neste artigo. À Profa. M. Z. C. Martins, agradeço pela cuidadosa revisão gramatical do texto.

\section{REFERÊNCIAS}

1. Cordell, G. A.; Phytochemistry 1995, 40, 1585.

2. Strege, M. A.; J. Chromatogr., B 1999, 725, 67.

3. Balandrin, M. F.; Klocke, J. A.; Wurtele, E. S.; Bollinger, W. H.; Science 1985, 228, 1154.

4. Buss, A. D. Em Bioassay Methods in Natural Product Research and Drug Development; Bohlin, L.; Bruhn, I. N., eds.; Kluwer Academic Publishers: Dordrecht, 1999.

5. Andrade, L. A. F.; Ciência e Cultura 1988, 40, 665.

6. Small, G. W.; Rabins, P. V.; Barry, P. P.; JAMA 1997, 278, 1363.

7. Shitani, E. Y.; Uchida, K. M.; Am. J. Health System Pharm. 1997, 54, 2805.

8. Bryne, G. J. A.; Aust. J. Hosp. Pharm. 1998, 28, 261.

9. Bierer, L. M.; Haroutunian, V.; Gabriel, S.; Knott, P. J.; Carlin, L. S.; Purohit, D. P.; Perl, D. P.; Philipkananof, J. S.; Davis, K. L.; J. Neurochem. 1995, 64, 749

10. Perry, E. K.; Pickering, A. T.; Wang, W. W.; Houghton, P. J.; Perry, N. S. L.; J. Pharm. Pharmacol. 1999, 51, 527.

11. Capone, G. T.; Mental Retardation and Developmental Disabilities Research Reviews 1998, 4, 36.

12. Hake, A. M.; Cleveland Clinic Journal of Medicine 2001, 68, 608.

13. Matos, F. J. A. Em Introdução à Fitoquímica Experimental; Edições UFC: Fortaleza, 1988, p. 39.

14. Espinola, E. B.; Dias, R. F.; Mattei, R.; Carlini, E. A.; J. Ethnopharmacol. 1997, 55, 223.

15. Bravo, J. A.; Sauvain, M.; Gimenez, A.; Munoz, V.; Callapa, J.; Le MenOlivier, L.; Massiot, G.; Lavaud, C.; Phytochemistry 1999, 50, 71.

16. Kang, S. Y.; Lee, K. Y.; Sung, S. H.; Park, M. J.; Kim, Y. C.; J. Nat. Prod. 2001, 64, 683.

17. Ellman, G. L.; Biochem. Pharmacol. 1961, 7, 88.

18. Rhee, I. K.; Meent, M.; Ingkaninan, K.; Verpoorte, R.; J. Chromatogr., A 2001, 915, 217. 